\author{
MILITARY TECHNICAL COLLEGE \\ CAIRO - EGYPT
}

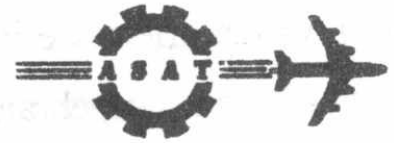

$7^{\text {th }}$ INTERNATIONAL CONF. ON AEROSPACE SCIENCES \& AVIATION TECHNOLOGY

\title{
Factors Affecting the Image Quality in the Computed Tomography Technique .
}
* A-N. Zayed
** B. Lawton

\begin{abstract}
The image quality, of an image reconstructed by a computed tomography (CT) system, is presented as a factor depending on the standard deviation of the reconstructed image. The main factors affecting the image quality are classified to three main groups depending on the CT system (hardware), the CT algorithm (software), and the scanning parameters. Each of these groups is studied, explained, and applied on a real scan. The images reconstructed of an object (model of a solid propellant rocket motor) by varying the type of detector, the collimator, the CT algorithm, the image resolution, and the scanning parameter are presented and compared by calculating the image quality factor.
\end{abstract}

\section{INTRODUCTION}

In the computed tomography (CT) technique, the image quality is represented by the spatial resolution, the contrast resolution, and the reconstructed values of the reconstructed variable. The spatial resolution is defined as the minimum resolvable distance between two objects. The contrast resolution is defined as the minimum detectable difference of the reconstructed variable. Sekihara ${ }^{[1]}$ developed a theoretical model to calculate the contrast-detail diagrams that can be used to express the image quality of an x-ray CT. The implemented CT system $[2,3]$ was used to scan and reconstruct images of an object (model of a solid propellant rocket motor) cross-section. This CT system consists of a gamma-ray source, radiation detector, movement system, pre-amplifier, main amplifier, low pass analogue filter, analogue-to-digital converter, and personal computer.

The main factors, which affect the image quality in the CT technique, are classified to three groups. The first group depends on the CT system (hardware), the second depends on the CT algorithm, and the third depends on the scanning parameters. The parameters, of the CT hardware, which affect the image quality are:

(a) sensitivity of the radiation detector(s),

(b) error in adjusting the projection angle,

(c) error in adjusting the ray sum position,

(d) ray sum (beam) thickness.

* Department of Rockets, Military Technical College, Kobry El-Kobba, Cairo, Egypt.

** School of Engineering and Applied Science, Royal Military College of Science, Shrivenham, England. 
The parameters, of the CT algorithm, which affect the image quality are:

(a) type of the reconstruction technique (Algebraic Reconstruction Technique "ART", Linear Super-position with Compensation "LSC", and the Fourier Convolution Technique "FCT"),

(b) by using the FCT, type of the used filter function,

(c) resolution of the reconstructed image.

The scanning parameters affecting the image quality are:
(a) number of projections
(b) number of ray sums

The images of the tested object reconstructed by different algorithms, scanning methods, and scanning parameters are illustrated and compared with the real mode. The comparison was made on the reconstructed variable by calculating the Image Quality Factor IQF which is created in this experimental study and defined as,

$$
I Q F \equiv 1-\sigma
$$

where $\sigma$ is the standard deviation of all the pixels in the scanning domain with respect to the real value. By representing the tested object as a matrix of NxN pixels, $\sigma$ is defined as,

$$
\sigma \equiv \sqrt{\frac{1}{N^{2}} \sum_{i=1}^{N} \sum_{j=1}^{N}\left(\frac{C h_{i, j}-h_{i, j}}{C h_{i, j}}\right)^{2}}
$$

where $h_{i, j}$ is the reconstructed value of a pixel in the $i^{\text {th }}$ row and the $j^{\text {th }}$ column, $\mathrm{Ch}_{\mathrm{i}, \mathrm{j}}$ is the real value of this pixel, and $\mathrm{N}$ is the order of the matrix which represents the tested object.

\section{CT SYSTEM}

The computed tomography systems, which have been used in the industrial applications [2-4], have different features of that which are used in the medical applications especially for the type of radiation, the scanning movement, and the scanning time. The implemented CT system [2,3], Fig. 1, was used to scan and reconstruct images of an object cross-section. The radiation source is a ${ }^{60} \mathrm{Co}$ gamma-ray source, the radiation detector is a Sodium Iodide $\mathrm{NaI}$ scintillation detector integrated with a photomultiplier tube. The signals collected from the detector are amplified by a pre-amplifier and main amplifier, filtered by a low-pass analogue filter, digitized by an analogue-to-digital converter, and stored in a personal computer. These data are used as input for the reconstruction software (CT algorithm) to reconstruct an image of the tested object cross-section at the scanning position. 
1. radiation source

2. radiation detector

3. pro-amplifier

4. main amplifier

5. low-pass analogue filter

6. analog-to-digital converter

7. personal computer

8. movement system

9. tested object

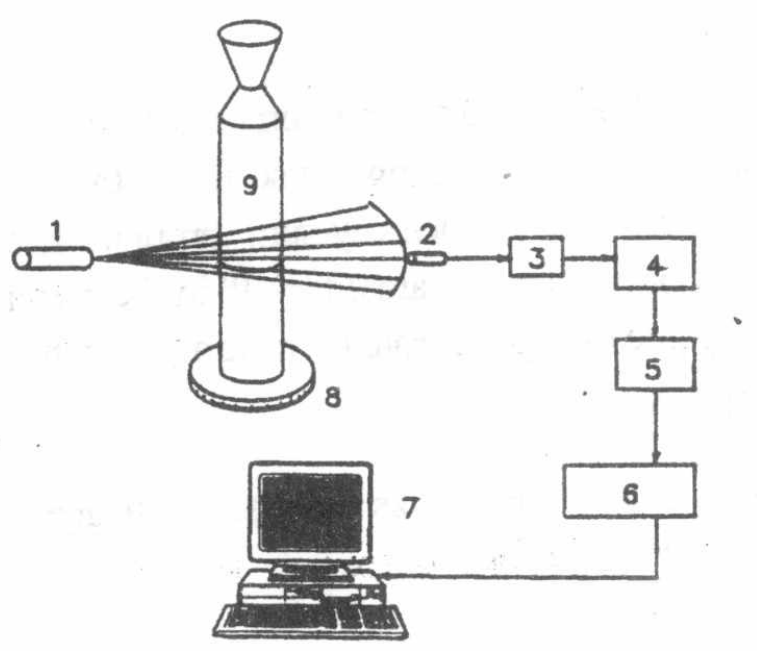

Fig. 1 Scheme of the implemented CT system

The tested object is a model of a solid propellant rocket motor. The model cross-section is shown in Fig. 2. The casing was made of steel $\left(\mathrm{h}_{\mathrm{St}}=0.0419 \mathrm{~mm}^{-1}\right)$ as a cylinder of $80 \mathrm{~mm}$ diameter and $4 \mathrm{~mm}$ thickness. The cross perforated grain was made of aluminium $\left(\mathrm{h}_{\mathrm{Al}}=\right.$ $0.0151 \mathrm{~mm}^{-1}$ ) of $20 \mathrm{~mm}$ core diameter, $10 \mathrm{~mm}$ ripe width, and $40 \mathrm{~mm}$ ripe length. Each ripe is ended by a semi-circle of $5 \mathrm{~mm}$ radius.

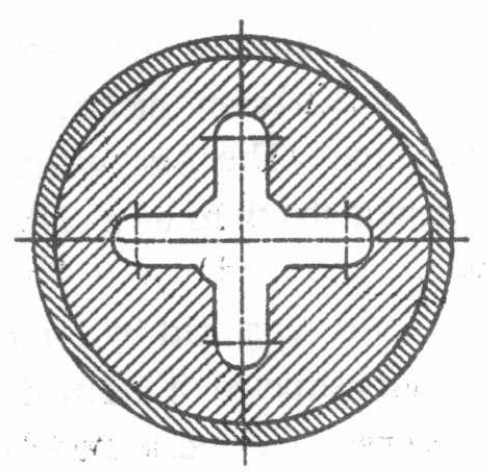

Fig. 2 Cross-section of the tested object (model of a solid propellant rocket motor)

\section{FACTORS, OF THE CT HARDWARE, AFFECTING THE IMAGE QUAUTY}

\subsection{Sensitivity of the Radiation Detector}

The measured value of the same attenuated ray sum is varying by using different types of radiation detectors [3], that is due to the sensitivity of each detector which affect the measured value of the ray sum and so the reconstructed image. The author [3] reconstructed two images of an educational model of a solid propellant rocket motor by the same scanning parameter. The first was by using a NaI detector which gives $\mathrm{IQF}=0.9833$. The second was by using the photo-diode (BPX 65) as a radiation detector which gives $\mathrm{IQF}=0.9698$. 


\subsection{Enror in Adjusting the Projection Angle}

The scanning limits of projections are $0: \pi$. The number of projections is determined by the angular shift between two projections. During the scanning by the implemented CT system, the system collects 12000 readings for each ray sum through the scanning from $0^{\circ}$ to $180^{\circ}$. This means that to use 180 projections separated by $1^{\circ}$ in the reconstruction, each projection is calculated by the mean value of approximate 67 readings which gives an average value and so decreases the image quality.

It is recommended to use a shaft encoder as an external trigger to collect the data at the specified position(s) of the projection(s).

\subsection{Error in Adjusting the Ray Sum Position}

Because of a lack in the fund, the scanning was made by using a single radiation detector. This detector is shifted through all the ray sums positions. In the case of the parallel beams scanning, the error was $\approx 0.2 \mathrm{~mm}$. In the fan-beam scanning, the error was $\approx 0.3^{\circ}$.

To avoid this errors, it is recommended to use a detector array which has pre-adjusted and accurate positions.

\subsection{The Scanning Beam Thickness}

The scanning beam thickness is represented by the detector collimator aperture. Ichihara[5] demonstrated the effect of the fan-beam collimator on the spatial resolution and the sensitivity of the imaging. To study the effect of the beam thickness on the image quality, the tested object, Fig. 2, was scanned three times. The first without collimator, the second by a collimator of $5 \mathrm{~mm}$ beam thickness, and the third by a collimator of $2 \mathrm{~mm}$ beam thickness.

The model was scanned by 81 ray sums separated by $1 \mathrm{~mm}$ and 72 projections $(0: \pi)$. The two- and three-dimensional images reconstructed of the model cross-section are shown in Fig. 3. The image quality factors IQFs were calculated for the three images as shown in Table.1 which shows the effect of the beam thickness on the image quality.

Table 1 IQF vs collimators

\begin{tabular}{|c|c|}
\hline Collimator & IQF \\
\hline No collimator & 0.9587 \\
\hline $5 \mathrm{~mm}$ & 0.9688 \\
\hline $2 \mathrm{~mm}$ & 0.9742 \\
\hline
\end{tabular}



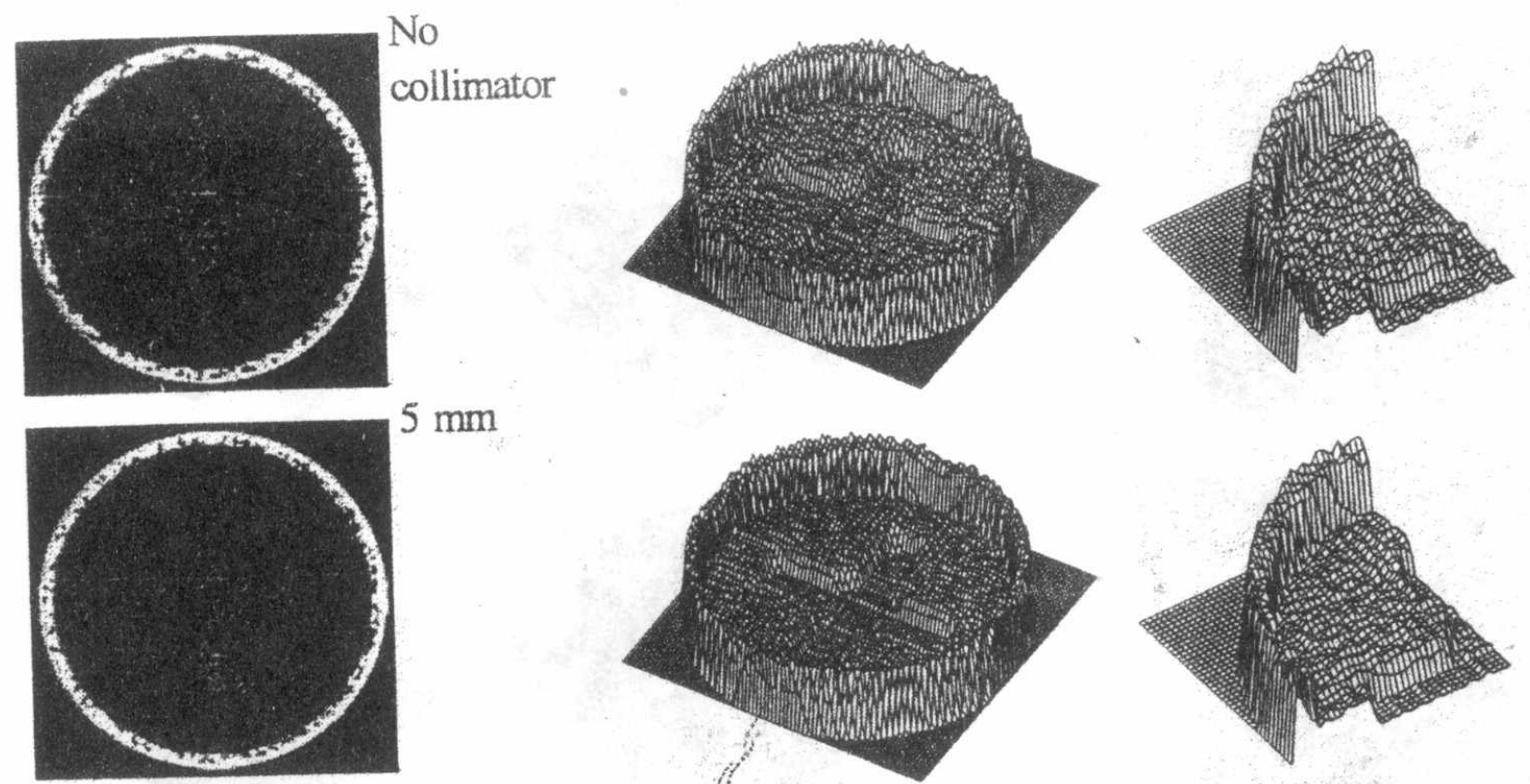

$5 \mathrm{~mm}$
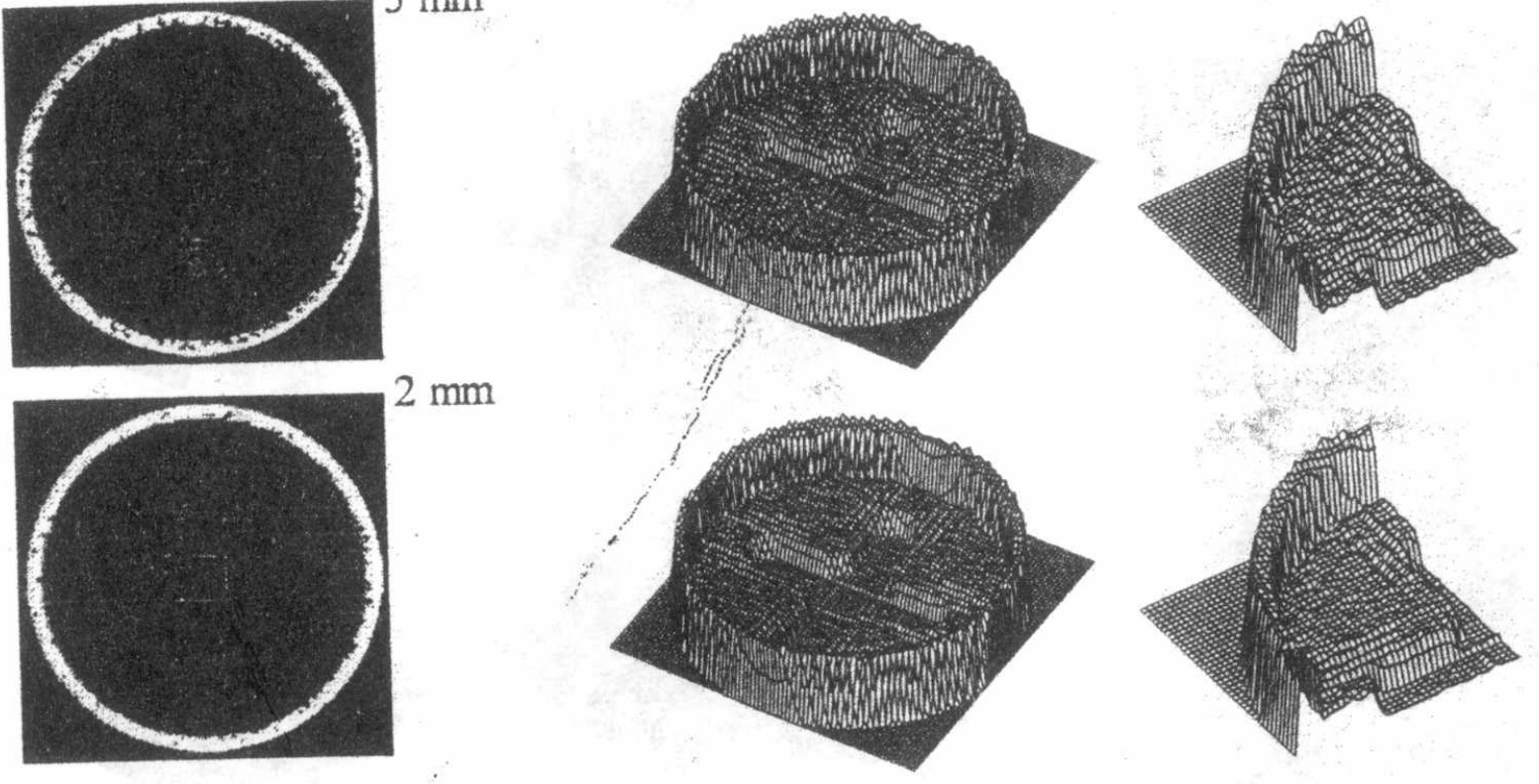

Fig. 3 Two- and three-dimensional, images reconstructed by (upper) no collimator, (middle) $5 \mathrm{~mm}$ beam thickness, and (lower) $2 \mathrm{~mm}$ beam thickness.

\section{FACTORS, OF THE CT SOFTWARE, AFFECTING THE IMAGE QUALTTY}

\subsection{The Reconstruction Technique}

Several CT algorithms have been used since the first generation scanner. The most familiar CT algorithms are the Algebraic Reconstruction Technique "ART", the Linear Superposition with Compensation "LSC", and the Fourier Convolution Technique "FCT". After the comparative study on the CT algorithms (ART, LSC, and FCT) had been made [6], it was found that the most precise algorithm is the FCT. To verify that conclusion, the measured data of the 81 ray sums through the 72 projections were used to reconstruct images of the rocket model by using the three algorithms. The two- and three-dimensional images reconstructed by the three algorithms are shown in Fig. 4. The IQFs of these images are shown in Table 2. 


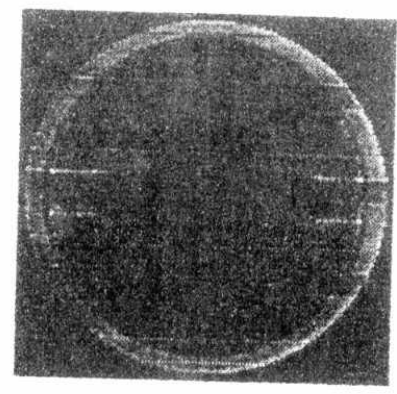

ART
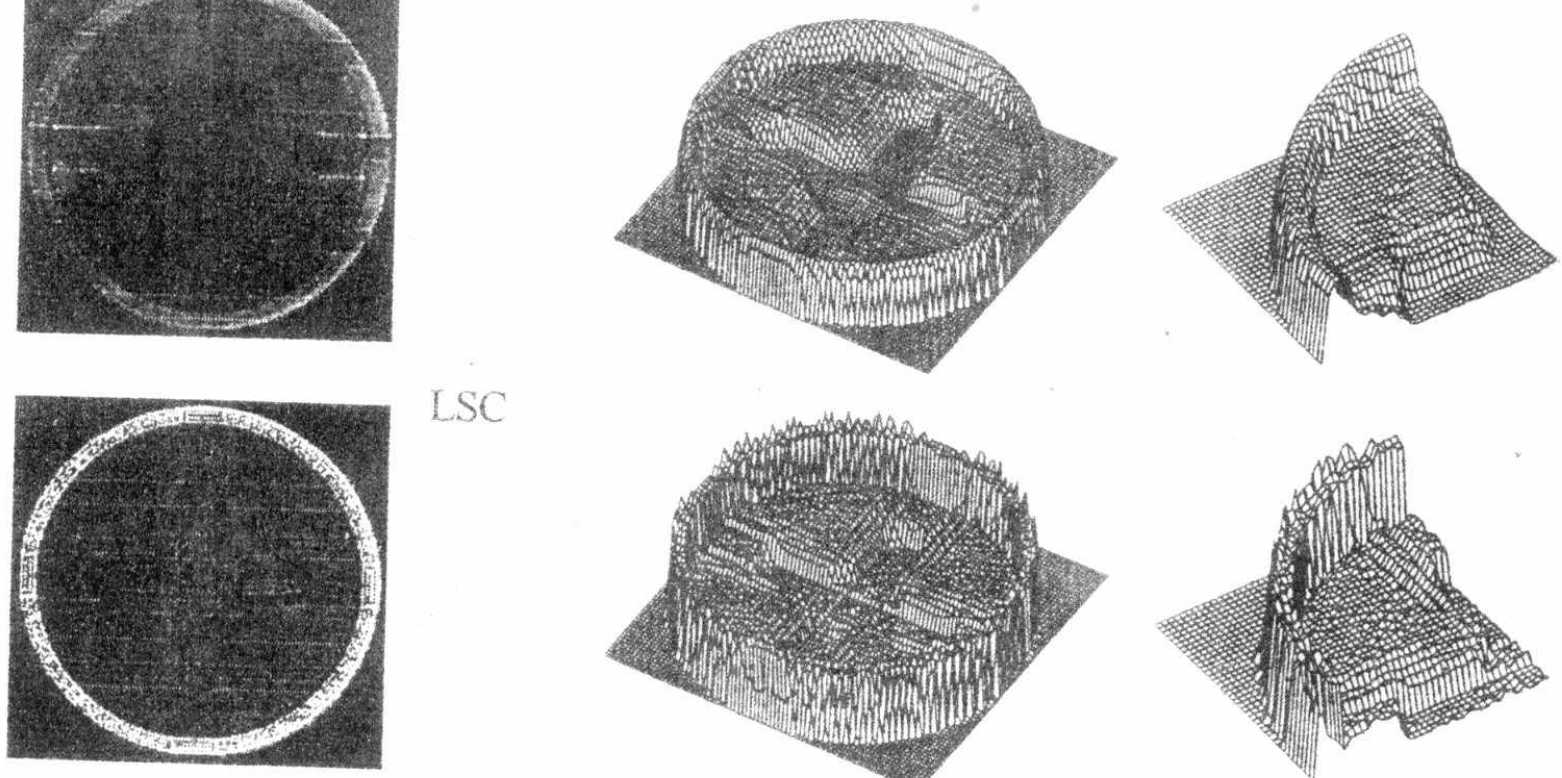

LSC
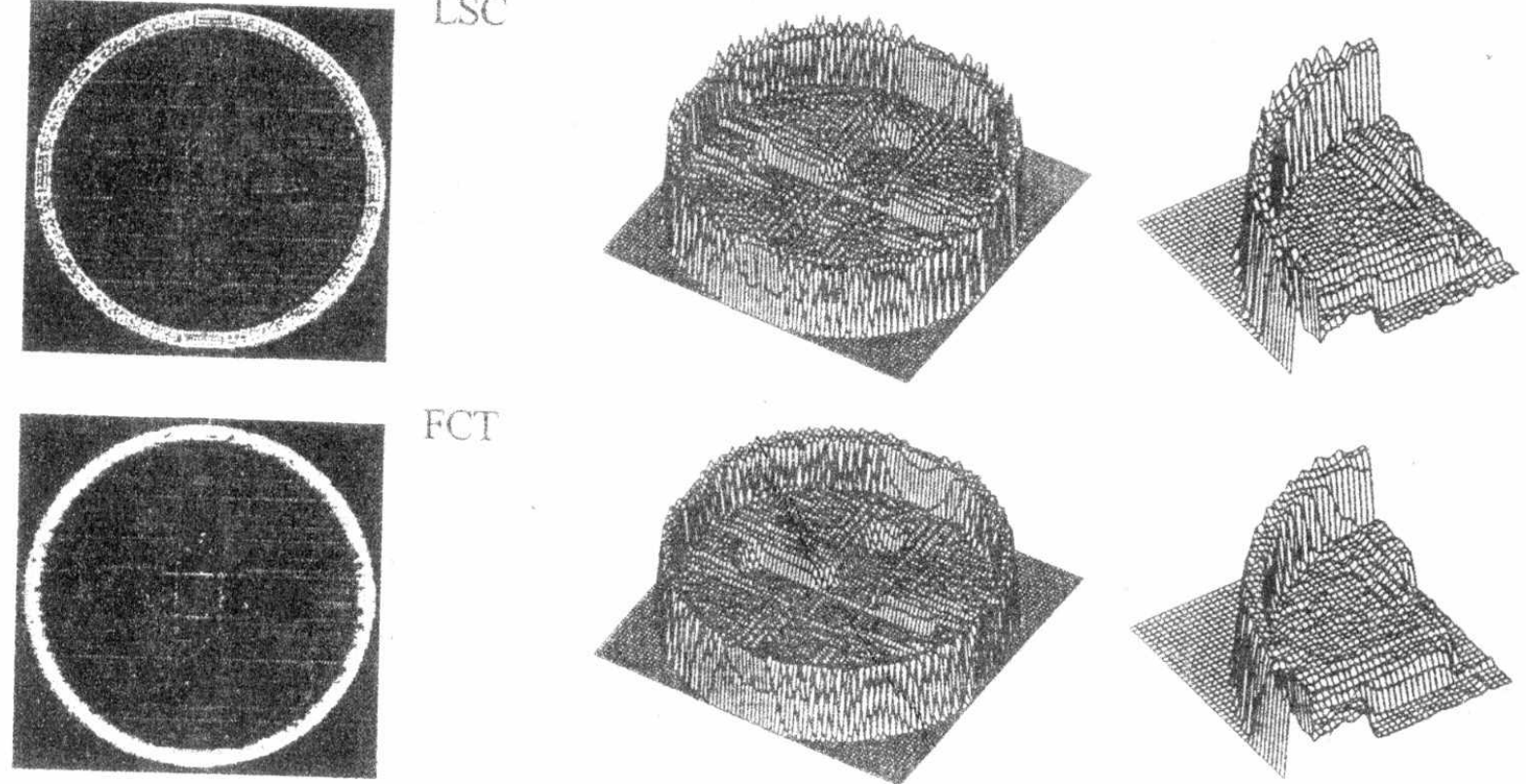

FCT
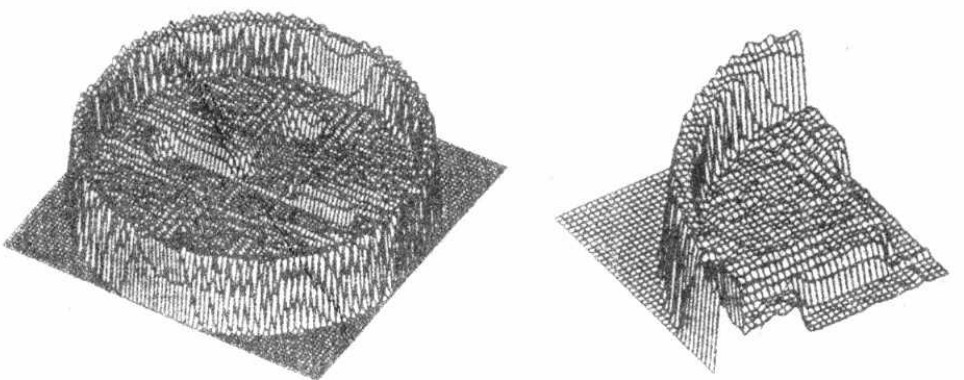

Fig. 4 Two- and three-dimensional images reconstructed by (upper) ART, (middle) LSC, anc
(lower) FCT.

Table 2 IQF vs reconstruction techniques

\begin{tabular}{|c|c|}
\hline algorithm & IQF \\
\hline \hline ART & 0.9268 \\
\hline LSC & 0.9591 \\
\hline FCT & 0.9742 \\
\hline
\end{tabular}

\subsection{By Using the FCT, the Used Filter Function}

In the reconstruction by using the FCT, Shepp and Logan [7] presented the reconstructed variable, $h$, from the Fourier inversion formula and transformed the integral form in this formula to an approximated summation depending on the filter function, $\Phi$, Shepp and Logan [8] modified this filter function to be $\Phi^{*}$ to decrease the effect of noise with some loss of the spatial resolution. The authors [9] presented a new filter function $\Phi^{* *}$ with a new version of the FCT. 
The images, of the tested object, reconstructed by using $\Phi, \Phi^{*}$, and the new version with $\Phi^{* *}$ are shown in Fig. 5 to show the effect of the filter function on the image quality. Table 3 shows IQFs of the images presented in Fig. 5.

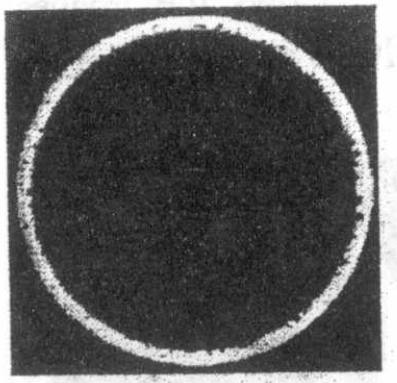

$\Phi$

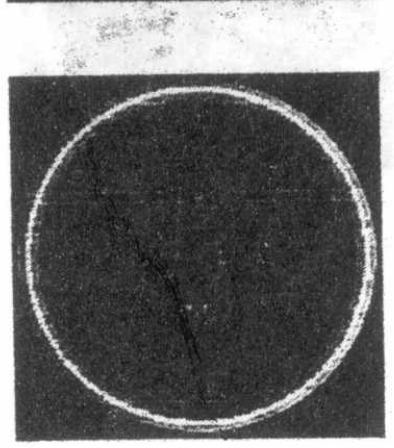

$\Phi$

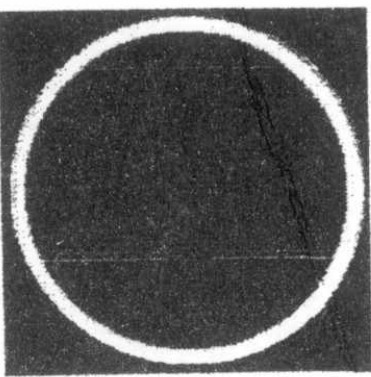

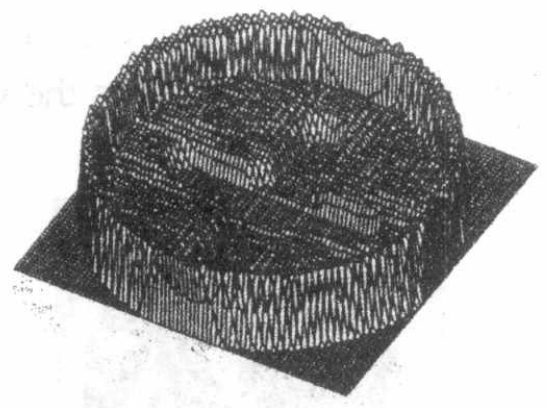
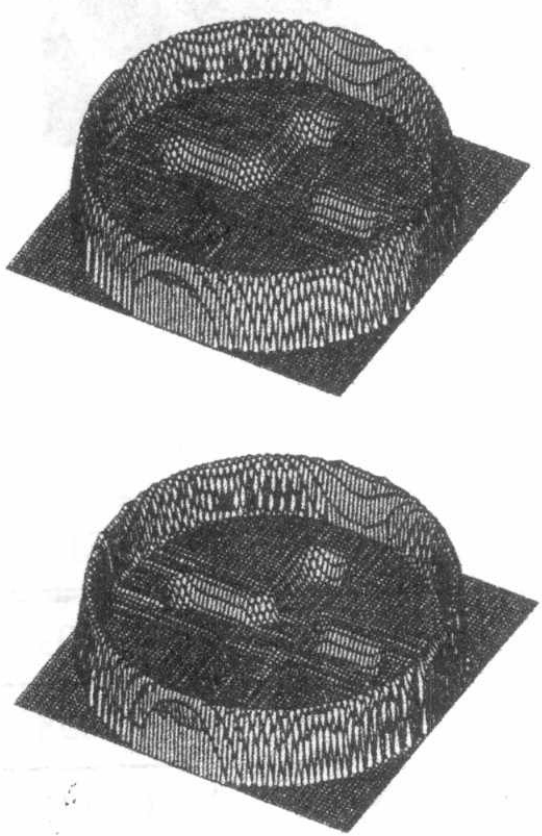
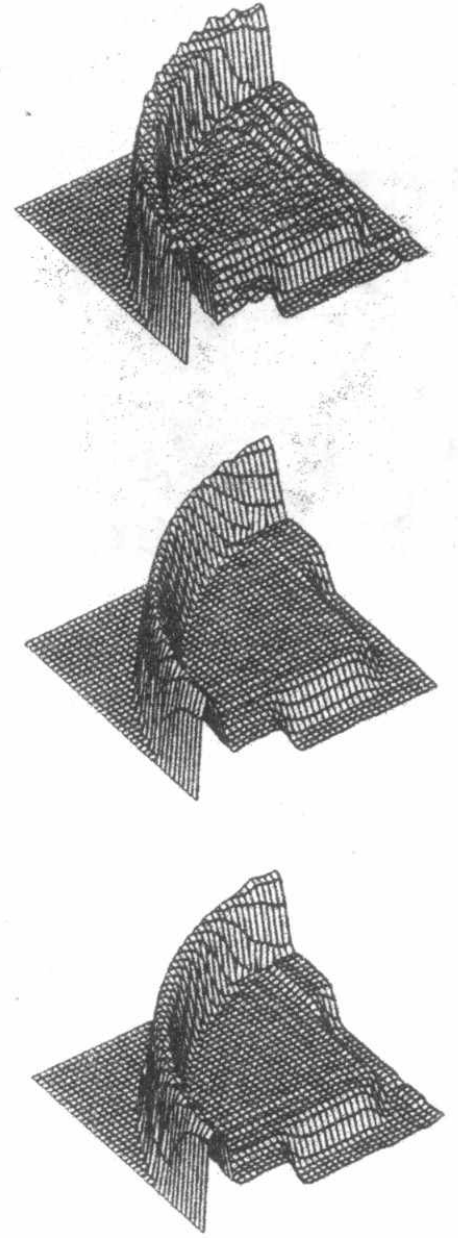

Fig. 5 Two- and Three-dimensional images reconstructed by using (upper) $\Phi$, (middle) $\Phi^{*}$, and (lower) the new version with the modified filter $\Phi^{* *}$

Table 3 IQF vs filter functions

\begin{tabular}{|c|c|}
\hline Filter function & IQF \\
\hline$\Phi$ & 0.9742 \\
\hline$\Phi^{*}$ & 0.9687 \\
\hline new version $+\Phi^{* *}$ & 0.9858 \\
\hline
\end{tabular}




\subsection{Resolution of the Reconstructed Image}

To show the effect of the resolution on the image quality, three images were reconstructed with different resolutions $(1 \mathrm{~mm}, 0.5 \mathrm{~mm}$, and $0.25 \mathrm{~mm}$ ). Fig. 6 shows the images reconstructed with different resolutions. Table 4 shows the values of IQFs of these images.
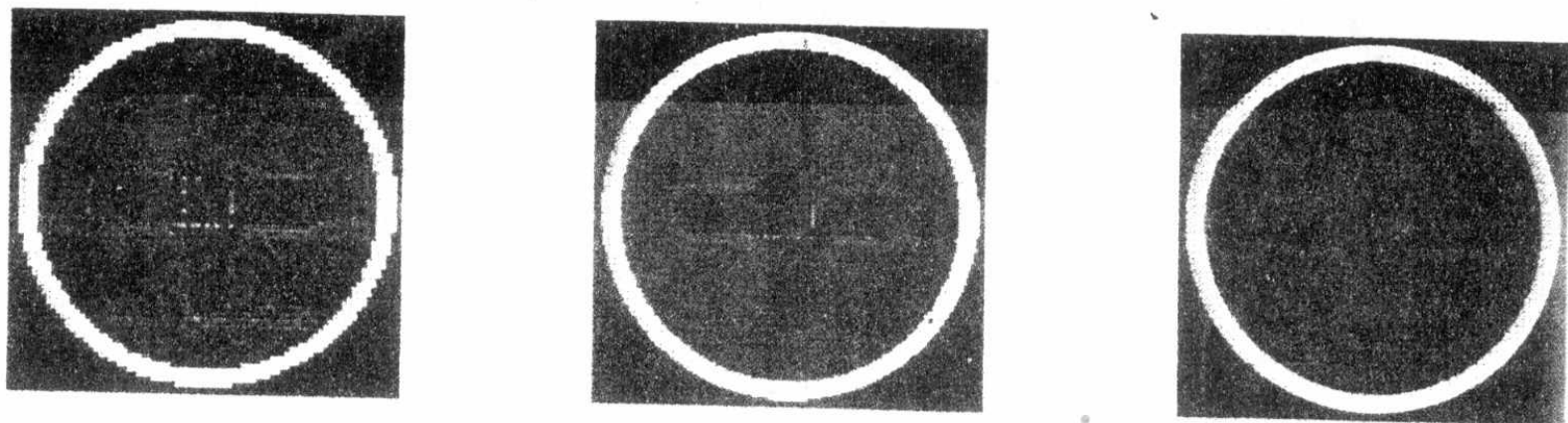

Fig. 6 Images reconstructed by different resolution (left) $1.0 \mathrm{~mm}$, (middle) $0.5 \mathrm{~mm}$, an (right) $0.25 \mathrm{~mm}$

Table 4 IQF vS resolutions

\begin{tabular}{|c|c|}
\hline Pixel size & IQF \\
\hline \hline $1.0 \mathrm{~mm}$ & 0.9793 \\
\hline $0.5 \mathrm{~mm}$ & 0.9858 \\
\hline $0.25 \mathrm{~mm}$ & 0.9871 \\
\hline
\end{tabular}

\section{SCANNING PARAMETERS AFFECTING THE IMAGE QUALTY}

The scanning parameters, which affect the image quality, are the number of ray sums and the number of projections. Series of images were reconstructed by different numbers of projections $M$ and different numbers of ray sums $K$ to show the effect of the number of projections and the number of ray sums on the image quality. Fig. 7 illustrates 9 images, each row was reconstructed by a different number of projections ( $M=18,36$, and 72 projections) and each column was reconstructed by a different number of ray sums $(K=21,41$, and 81 ray sums). The IQFs of these images are recorded in Table 5 to show the effect of the scanning parameters on the image quality. The scanning method (parallel beams scanning or fan-beam scanning) also affects the image quality [6] .. In the case of the fan-beam scanning, the images reconstructed by different fan-beams (different fan vertex angle) have different image quality. The effect of the scanning method and the fan vertex angle are not included in this study. 

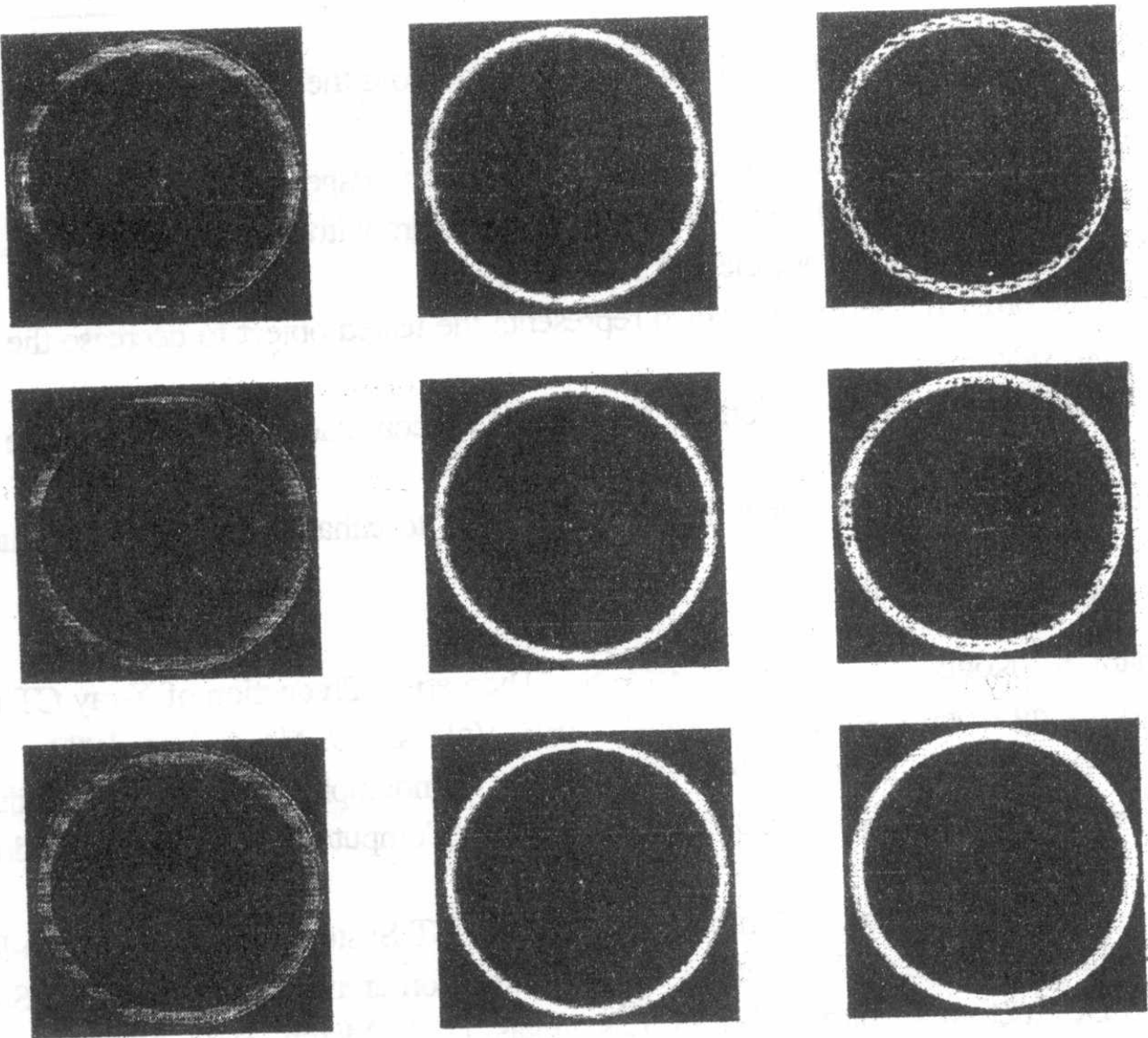

Fig. 7 Images reconstructed by different scanning parameters (upper) $M=18$, (middle) $\mathrm{M}=36$, (lower) $\mathrm{M}=72$ projections, (left) $\mathrm{K}=21$, (middle) $\mathrm{K}=41$, and (right) $\mathrm{K}=81$ ray sums

Table 5 IQF vs $M$ and $K$

\begin{tabular}{|c|c|c|c|}
\hline \multirow{2}{*}{$M$} & \multicolumn{3}{|c|}{$\mathrm{K}$} \\
\cline { 2 - 4 } & 21 & 41 & 81 \\
\hline 18 & 0.9379 & 0.9556 & 0.9681 \\
\hline 36 & 0.9392 & 0.9587 & 0.9738 \\
\hline 72 & 0.9411 & 0.9632 & 0.9858 \\
\hline
\end{tabular}

\section{CONCLUSION}

The image quality, of an image reconstructed by a CT system, can be indicated by calculating the image quality factor IQF which represents the spatial resolution and the reconstructed values of the reconstructed variable. To get the highest IQF (very close to the value of one), the following recommendations are required:

(a) the radiation detector of a higher sensitivity decreases the error in the scanning data,

(b) the positions of projections have to be determined by using a shaft encoder as an externa trigger to avoid the error of the projections positions, 
(c) use a detector array to contain all the detectors and avoid the error in the scanning beam
position,

(d) the narrower scanning beam thickness gives the better image quality,

(e) use the new version of the Fourier convolution algorithm with the modified filter function to reconstruct the image from the scanning data,

(f) increase the order of the matrix which represents the tested object to decrease the image resolution (pixel size), (g) increase the number of projections to get the pixel reconstructed value very close to the
real value, and

(h) increase the number of ray sums, in each projection, to enhance the spatial resolution.

\section{REFERENCES}

[1] Sekihara, K., Kohno, H., and Yamamoto, S., "Theoretical Predection of X-ray CT image Quality Using Contrast-Detail Diagram", IEEE, Vol. NS-29, No. 6, Dec. 1982.

[2] Zayed, A-N., and Lawton, B., "Computed Tomography System for Industrial Application", Proceeding of the 3rd IASTED Conf. (Computer Applications in Industry), Cairo, Dec. 26-29, 1994.

[3] Zayed, A-N., and Lawton, B.,"Implementation of a CT System by Using a Photo-Diode as a Radiation Detector", Proceeding of the Spring Conference of the American Society of Non-Destructive Testing ASNT95, Las Vegas, USA, March 20-23, 1995.

[4] MacCuaig, N., "The Industrial Application of Low Cost Gamma-ray Tomography". Biritch J. of Non-destructive Test, Vol. , 1992

[5] Ichihara, T., and Nambu, K, "High Spatial Resolution Reconstruction Technique for SPECT Using a Fan-Beam Collimator", IEEE, Vol. NS-40, No. 4, Aug. 1993.

[6] Zayed, A-N., and Lawton, B., "Comparative Study on Computed Tomography Algorithms", Proceeding of SPIE '94 (Visual Communication and Image Processing), Chicago, USA, Sept. 1994.

[7] Shepp, L.A., and Logan, B.F., "Reconstructing Interior Head Tissue From X-Ray Transmissions", IEEE, Vol.NS-21, No. 1, Feb. 1974

[8] Shepp, L.A., and Logan, B.F., "The Fourier Reconstruction of a Head Section", IEEE, Vol. NS-21, No. 3, June 1974.

[9] Zayed, A-N. and Lawton, B., "A New Version of the Fourier Convolution Algorithm in the CT Technique", Proceeding of SPIE '94 (Visual Communication and Image Processing), Chicago, USA, Sept. 1994. 